\title{
Frequency chirp and pulse shape effects in self-modulated laser wakefield accelerators*
}

\author{
C. B. Schroeder, ${ }^{\dagger}$ E. Esarey, C. G. R. Geddes, Cs. Tóth, B. A. Shadwick, J. van Tilborg, ${ }^{\ddagger}$ J. Faure, and W. P. Leemans \\ Lawrence Berkeley National Laboratory, University of California, Berkeley, California 94720
}

\begin{abstract}
The effect of asymmetric laser pulses on plasma wave excitation in a self-modulated laser wakefield accelerator is examined. Laser pulse shape and frequency chirp asymmetries, controlled experimentally in the laser system through a grating pair compressor, are shown to strongly enhance measured electron yields for certain asymmetries. It is shown analytically that a positive (negative) frequency chirp enhances (suppresses) the growth rate of the Raman forward scattering and near-forward Raman sidescatter instabilities, but is of minimal importance for the experimental parameters. Temporal laser pulse shapes with fast rise times ( $\lesssim$ plasma period) are shown to generate larger wakes (compared to slow rise time pulses) which seed the growth of the plasma wave, resulting in enhanced electron yield.
\end{abstract}

PACS numbers: 52.38.Kd, 52.38.-r, 52.38.Bv, 42.65.Re

\section{INTRODUCTION}

Controlling the coupling of laser light to plasma waves is important in numerous applications, such as laser-plasma accelerators, ${ }^{1}$ laser-plasma-based harmonic generation,${ }^{2}$ x-ray lasers, ${ }^{3}$ and laser-driven inertial confinement fusion schemes. ${ }^{4}$ Laser-plasma accelerators, which have been proposed as compact next-generation accelerators, rely on laser excitation of large amplitude plasma waves for trapping and acceleration of charged particles. ${ }^{1}$ The use of laser bandwidth, ${ }^{5,6}$ coherence, ${ }^{7}$ frequency chirp, ${ }^{8}$ and pulse shaping ${ }^{9,10}$ have been proposed as methods for controlling the laser-plasma coupling.

Raman forward scattering ${ }^{11}$ (RFS) can be used to drive the self-modulated laser wakefield accelerator (SM-LWFA), in which a long (compared to the plasma wavelength) laser pulse becomes modulated and produces a large amplitude plasma wave with phase velocity near the speed of light (for a review, see Ref. 1). This plasma wave, with 10$100 \mathrm{GeV} / \mathrm{m}$ accelerating gradients having been demonstrated using present laser technology, can be used to accelerate charged particles to high energies. ${ }^{12-17}$ Recent experiments by Faure et al. ${ }^{18}$ have studied the dynamics of RFS using picosecond linearly chirped pulses and found no dependence on the sign of the chirp for laser intensities $~$ $2 \times 10^{17} \mathrm{~W} / \mathrm{cm}^{2}$. Other experiments by Yau et al. ${ }^{19}$ have reported enhanced efficiency of the RFS instability for positively-chirped laser pulses.

In this paper, we discuss recent experiments conducted using the short pulse, high peak power and high repetition rate Ti: $\mathrm{Al}_{2} \mathrm{O}_{3}$ laser system ${ }^{17}$ at Lawrence Berkeley National Laboratory (LBNL), which reported frequency chirp induced asymmetries in the SM-LWFA electron and neutron yields. ${ }^{20}$ To interpret these measurements we theoretically examine the influence of asymmetries in frequency chirp and temporal pulse shape on the laser-excitation of plasma waves. Section II describes the LBNL SM-LWFA experimental results. In Sec. III, we present a calculation of the effects of a frequency chirp on the growth of the RFS and near-forward Raman sidescatter (RSS) instabilities, which are responsible for the generation of high phase velocity plasma waves. In Sec. IV, we examine the seed generated by asymmetric temporal pulse shapes. Section V summarizes the results presented in this paper and discusses the implications for future experiments.

\section{EXPERIMENTAL OBSERVATIONS}

In the SM-LWFA experiments preformed at LBNL, ${ }^{20}$ strong asymmetry in the yield of energetic electrons (and neutron production) was observed as a function of compressor setting (which corresponds to variations in pulse duration, frequency chirp, and pulse shape). In these experiments, low energy laser pulses (of wavelength $\lambda \simeq 0.8 \mu \mathrm{m}$ ) from a Ti: $\mathrm{Al}_{2} \mathrm{O}_{3}$ laser oscillator were first temporally stretched, amplified to $1 \mathrm{~J} /$ pulse level, and then compressed using a grating based optical compressor. Following compression, the laser beam was focused to a $6 \mu \mathrm{m}$ spot size (Rayleigh length $\left.Z_{R} \simeq 0.14 \mathrm{~mm}\right)$ with a $30 \mathrm{~cm}$ focal length $(\mathrm{F} / 4)$ off-axis parabola onto a pulsed gas jet. The peak power $P$ of the laser was varied using both the pulse duration and laser energy. At optimum compression $[55$ fs full-width-halfmaximum (FWHM) duration], $P \simeq 8.3 \mathrm{TW}$, resulting in a calculated peak intensity $I=2 P / \pi r_{s}^{2} \simeq 1.5 \times 10^{19} \mathrm{~W} / \mathrm{cm}^{2}$ and a normalized laser strength $a_{0} \simeq 8.6 \times 10^{-10} \lambda[\mu \mathrm{m}] I^{1 / 2}\left[\mathrm{~W} / \mathrm{cm}^{2}\right] \simeq 2.6$. The laser pulse spectral bandwidth was typically $21-22 \mathrm{~nm}$ FWHM, or $\Delta \omega / \omega_{0} \simeq 2.6 \%$.

The laser pulse duration, frequency chirp, and temporal envelope characteristics were varied by scanning the compressor grating separation. The laser pulse duration was measured with a single shot autocorrelator, located outside 


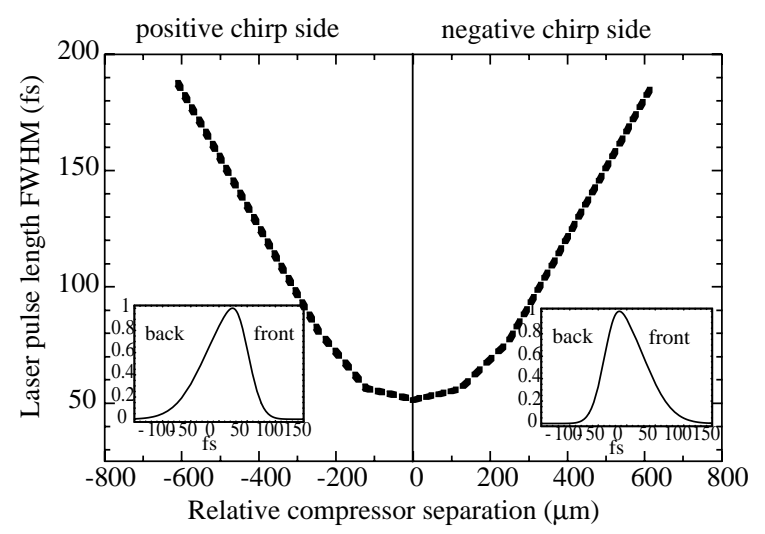

FIG. 1: Pulse duration measured from single shot autocorrelator (dashed line) vs. compressor setting. The insets show the normalized intensity temporal distributions $I(t) / I_{0}$ at two relative compressor separations corresponding to positively and negatively skewed pulses $(b \simeq \pm 0.6)$ of 76 fs FWHM duration.

the vacuum chamber, and temporally resolved spectra of the laser were measured using a frequency resolved optical gating (FROG) system, allowing pulse shape reconstruction. Figure 1 shows the pulse duration versus compressor setting. A frequency chirp was produced by translating one of the gratings of the compressor away from the optimum compression setting. Higher-order phase dispersive terms associated with optical components in the laser system will modify the temporal pulse shape. Pulse shapes for positively and negatively chirped pulses with 76 fs FWHM duration, are shown in Fig. 1. As can be seen in Fig. 1, accumulated phase nonlinearities ${ }^{21}$ from propagation through the laser system (including the compressor) resulted in positively (negatively) chirped pulses having a fast (slow) rise time. The experimental temporal pulse shapes can be well-fitted to a skewed-Gaussian of the form $I(t)=I_{0} \exp \left\{\left[-t^{2} /\left(2 \sigma_{t}^{2}\right)\right]\left[1+b t /\left(t^{2}+\sigma_{t}^{2}\right)^{1 / 2}\right]^{-1}\right\}$, where $b$ is the skew parameter and $\sigma_{t}$ is the pulse duration. It should be noted that, in principle, positively chirped pulses can also be generated with slow rise and fast fall times through different compressor tuning.

Typical experimental plasma profiles had peak density in excess of $3 \times 10^{19} \mathrm{~cm}^{-3}$ over $700 \mu \mathrm{m}$, with a $500 \mu \mathrm{m}$ ramp from vacuum on either side, as measured interferometrically. The minimum power at which electrons were observed was $\sim 0.9 \mathrm{TW}$ (pulse duration $\sim 500 \mathrm{fs}$ ), which is comparable to the critical power for relativistic self-guiding, ${ }^{22}$ $P_{\text {crit }}[\mathrm{GW}] \simeq 17.4\left(\lambda_{p} / \lambda\right)^{2}$, for an equilibrium plasma density $n_{0} \simeq 10^{19} \mathrm{~cm}^{-3}$ (plasma wavelength $\left.\lambda_{p} \simeq 11 \mu \mathrm{m}\right)$, where $\lambda_{p}=2 \pi c / \omega_{p}$ and $\omega_{p}^{2}=4 \pi e^{2} n_{0} / m$. Without guiding, the laser-plasma interaction is limited to about a Rayleigh length owing to diffraction. Experimental evidence for relativistic self-guiding of laser pulses above the critical power was observed in the transmitted laser spectra, shown in Fig. 2. Since the laser pulse is responsible for plasma creation through ionization of the He gas, frequency blueshifting in the laser pulse is observed due to the interaction with the comoving ionization front. For self-guided laser pulses, the laser intensity will exceed the ionization appearance intensity of $\mathrm{He}^{++}$by two orders of magnitude and hence only the front of the laser pulse will experience blueshifting. For laser pulses below the critical power, diffraction will reduce the laser intensity, resulting in a significant portion of the laser pulse undergoing frequency blueshifting. For compressor settings such that $P<P_{\text {crit }}$, the transmitted laser spectra contained significant blueshifting over the laser pulse. For compressor settings such that $P \gtrsim P_{\text {crit }}$ the amount of ionization blueshifting was drastically reduced (see Fig. 2), indicating guiding and resulting in electron production. When electrons were produced, spectra showed a clear redshifted sideband, which is attributed to RFS or near-forward RSS.

The dependence of electron yield on focal position within the plasma density profile was examined. The total charge per bunch of the electron beam was measured using an integrating current transformer. Two cases are shown in Fig. 3. In case 1, the laser was focused on the upstream edge (low density $\sim 2 \times 10^{18} \mathrm{~cm}^{-3}$ ) of the gas jet. In case 2 , the laser was focused into the jet at a density $\sim 3 \times 10^{19} \mathrm{~cm}^{-3}$. For case 2 , the peak plasma density was $25 \%$ lower, which is a small correction compared to the difference between the densities at the two focal locations.

For case 1, the maximum electron yield did not occur for the shortest laser pulse duration (defining the zero-setting of the compressor) and was found to be larger for positive chirp by about a factor 2 compared to negative chirp. In some runs, order of magnitude asymmetries have been observed in the electron data. For case 2, significantly less asymmetry was seen and the yield was found to peak close to the compressor zero. Although the peak yield is nearly equal in the two cases, focusing on the gas jet edge (case 1) produces significantly larger amounts of high energy electrons, as previously shown through gamma-neutron activation experiments. ${ }^{17}$

Several possible mechanisms have been investigated to explain the asymmetry in yield with compressor setting (i.e., 


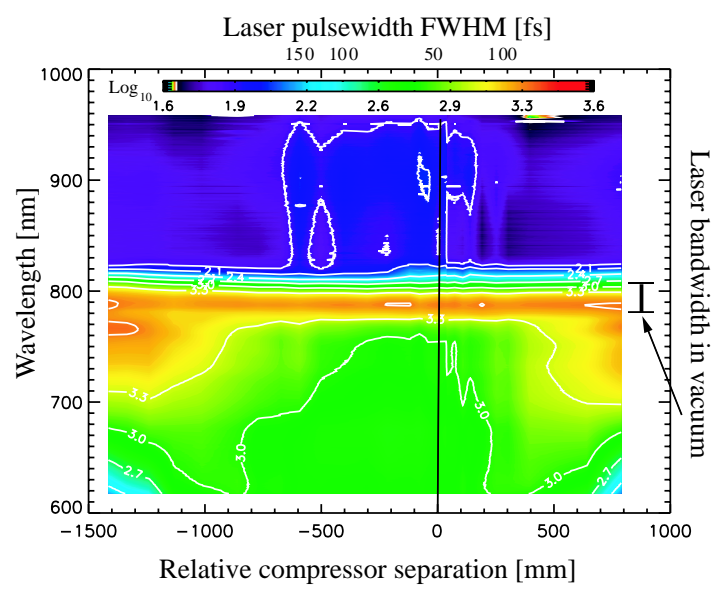

FIG. 2: Forward laser spectra vs. compressor setting for $\sim 460 \mathrm{~mJ} /$ pulse laser (duration $\geq 50$ fs) focused on the gas jet upstream edge.

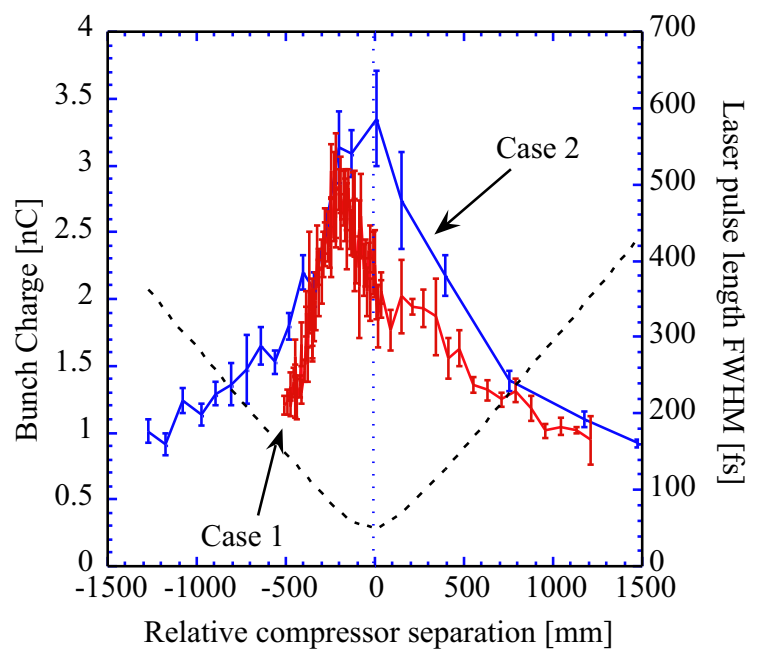

FIG. 3: Electron yield for cases 1 (red curve) and 2 (blue curve) and pulse duration (dashed line) vs. compressor setting. Asymmetry in electron yield is observed vs. compressor setting for case 1 .

positive and negative chirp for a fixed pulse length). In Sec. III, we examine the effects of a frequency chirp on RFS and near-forward RSS, which are responsible for high phase velocity plasma wave generation. In Sec. IV, we examine the seeding of the Raman instability by asymmetric pulse shapes.

\section{RAMAN SCATTERING OF CHIRPED LASER PULSES}

The effect of a finite-bandwidth on parametric instabilities, such as the Raman instability, has been extensively studied for the case of an uncorrelated, or random, frequency bandwidth. ${ }^{5,6}$ In this section we examine the effect of a correlated frequency chirp on the Raman instability growth rates.

To study the effects of a frequency chirp on RFS and near-forward RSS, we consider a Maxwell-fluid model of the laser-plasma interaction. In the Coulomb gauge, the Maxwell equations for the fields can be expressed as

$$
\begin{gathered}
{\left[\nabla^{2}-\frac{\partial^{2}}{\partial(c t)^{2}}\right] \vec{a}=k_{p}^{2} \frac{n}{n_{0}} \vec{\beta}+\frac{\partial}{\partial c t} \nabla \phi,} \\
\nabla^{2} \phi=k_{p}^{2}\left(\frac{n}{n_{0}}-1\right),
\end{gathered}
$$

where $k_{p}=2 \pi / \lambda_{p}, \vec{a}$ and $\phi$ are the transverse vector and scalar potentials, respectively, normalized to $m c^{2} / e$, and 
$n$ is the plasma number density. The relativistic Lorentz factor is $\gamma=(1-\vec{\beta} \cdot \vec{\beta})^{-1 / 2}$, where $\vec{\beta}$ is the electron fluid velocity normalized to the speed of light.

A cold-fluid model of the neutral plasma is assumed. Thermal effects may be ignored when the quiver velocity is much greater than the electron thermal velocity and the thermal energy spread is sufficiently small such that electron trapping in the plasma does not take place. The ions are also assumed to be stationary, which is typically the case for short-pulse $(\lesssim 1 \mathrm{ps})$ laser interactions in underdense plasmas. The cold fluid equations can be expressed as

$$
\begin{gathered}
\frac{\partial}{\partial c t}(\vec{\beta}-\vec{a})=\nabla(\phi-\gamma), \\
\frac{\partial n}{\partial c t}+\nabla \cdot(n \vec{\beta})=0,
\end{gathered}
$$

where $\nabla \times(\gamma \vec{\beta}-\vec{a})=0$ initially (before the passage of the laser pulse) was assumed.

In this work we will focus our analysis on laser propagation in an underdense plasma with group velocity of the laser approximately the speed of light. This allows one to make a time scale separation between fast (laser frequency) and slow (plasma frequency) time scales. ${ }^{23}$ The term $\partial_{c t} \nabla \phi$ can be neglected in Eq. (1) since the fast part of the electrostatic potential is typically small compared to the fast part of the plasma current, which is approximately the transverse vector potential $\gamma \beta_{\perp} \simeq a_{\perp}$ for an initially quiescent plasma.

To study the growth of Raman instabilities, we consider a density perturbation $\delta n=n / n_{0}-1$, which results from the scattering of a large-amplitude pump laser pulse $a_{\text {pump }}$ into daughter waves $a_{\text {scat }}$, such that $\left|a_{\text {pump }}\right| \gg\left|a_{\text {scat }}\right|$. Linearizing about the perturbations $\delta n$ and $a_{\text {scat }}$, Eqs. (1)-(4) can be combined to yield

$$
\left[\frac{\partial^{2}}{\partial c^{2} t^{2}}+\frac{k_{p}^{2}}{\gamma_{\perp 0}}\right] \delta n=\frac{1}{\gamma_{\perp 0}^{2}} \nabla^{2}\left(a_{\text {pump }} a_{\text {scat }}\right)
$$

and

$$
\left[\nabla^{2}-\frac{\partial^{2}}{\partial c^{2} t^{2}}-\frac{k_{p}^{2}}{\gamma_{\perp 0}}\right] a_{\text {scat }}=\frac{k_{p}^{2}}{\gamma_{\perp 0}} a_{\text {pump }}\left[\delta n-\frac{1}{\gamma_{\perp 0}^{2}}\left(a_{\text {pump }} a_{\text {scat }}\right)\right] .
$$

Here $\gamma_{\perp 0}^{2}=1+\left\langle a_{\text {pump }}^{2}\right\rangle$, where the angular-brackets denote a time-average over the fast laser period, and $k_{p} c / \gamma_{\perp 0}^{1 / 2}$ is the plasma frequency in the lab frame including the correction due to the relativistic quiver motion. It is convenient to work in the comoving variable $\zeta=z-c t$ and transform from the variables $(z, t)$ to $(\zeta=z-c t, \tau=c t)$. We will assume that the head of the right-going laser pulse is initially at $\zeta=0$ and the body of the laser pulse extends into the region $\zeta \leq 0$, while the plasma is unperturbed in the region $\zeta>0$. In the comoving variables $(\zeta, \tau)$ the linearized equations become

$$
\left[\left(\frac{\partial}{\partial \tau}-\frac{\partial}{\partial \zeta}\right)^{2}+\frac{k_{p}^{2}}{\gamma_{\perp 0}}\right] \delta \rho=\left(\nabla_{\perp}^{2}+2 \frac{\partial^{2}}{\partial \zeta \partial \tau}-\frac{\partial^{2}}{\partial \tau^{2}}-\frac{k_{p}^{2}}{\gamma_{\perp 0}}\right) \frac{a_{\text {pump }} a_{\text {scat }}}{\gamma_{\perp 0}^{2}}
$$

and

$$
\left(\nabla_{\perp}^{2}+2 \frac{\partial^{2}}{\partial \zeta \partial \tau}-\frac{\partial^{2}}{\partial \tau^{2}}-\frac{k_{p}^{2}}{\gamma_{\perp 0}}\right) a_{\text {scat }}=\frac{k_{p}^{2}}{\gamma_{\perp 0}} a_{\text {pump }} \delta \rho .
$$

where $\delta \rho=\delta n-a_{\text {pump }} a_{\text {scat }} / \gamma_{\perp 0}^{2}$.

We model the pump and scattered laser pulse normalized transverse vector potentials (linear polarization is assumed) as

$$
a_{\perp}=a_{\text {pump }}+a_{\text {scat }}=\frac{a_{0}}{2} e^{i \varphi_{0}}+\sum_{ \pm} \frac{a_{ \pm}}{2} e^{i \varphi_{ \pm}}+\text {c.c. }
$$

where $a_{0}$ is the pump amplitude, and $a_{ \pm}$are the slowly-varying envelopes of the Stokes (down-shifted) and anti-Stokes (up-shifted) scattered light waves. Note that, for direct RFS or near-forward RSS, both the Stokes and anti-Stokes waves can be approximately resonant. We will assume $\left|a_{0}\right| \gg\left|a_{ \pm}\right|$and $a_{0}$ is a nonevolving envelope (i.e., pump depletion effects are neglected). To isolate the effect of a frequency chirp we will consider laser pulses such that $k_{p} c \sigma_{t} \gg 1$, and the laser pulse vector potential amplitude is approximately uniform within the pulse. To lowest-order, $\left\langle a^{2}\right\rangle \simeq\left\langle a_{\text {pump }}^{2}\right\rangle=\left|a_{0}\right|^{2} / 2$. The temporal and spatial derivatives of the phase determine the local values of the pulse 
frequency and wave number for the pump (subscript 0) and scattered (subscript \pm ) light waves: $\omega_{0, \pm}=-\partial \varphi_{0, \pm} / \partial t$ and $\vec{k}_{0, \pm}=\nabla \varphi_{0, \pm}$. The plasma density perturbation is modeled as

$$
\delta \rho=\frac{\hat{\rho}}{2} e^{i \varphi_{p}}+\text { c.c. },
$$

where $\hat{\rho}$ is the slowly-varying envelope of the plasma density perturbation and $\varphi_{p}=k z+k_{\perp} x_{\perp}-\omega t$. The resonance condition for Raman scattering requires $\varphi_{ \pm}=\varphi_{0} \pm \varphi_{p}$. Note that here we are considering "whole-beam" instabilities such that $k_{\perp} \sim r_{s}^{-1}$, where $r_{s}$ is the laser spot size, and we model the scattered light and plasma waves as plane waves: $a_{ \pm}(\zeta, \tau)$ and $\hat{\rho}(\zeta, \tau)$.

Using the eikonal (slowly-varying envelope) approximation $\left|\partial_{\tau} \hat{\rho}\right| \ll|k \hat{\rho}| \sim|\omega \hat{\rho} / c|,\left|\partial_{\tau} a_{ \pm}\right| \ll\left|k a_{ \pm}\right| \sim\left|\omega a_{ \pm} / c\right|$, and $\left|\partial_{\zeta} a_{ \pm}\right| \ll\left|k a_{ \pm}\right| \sim\left|\omega a_{ \pm} / c\right|$, Eq. (7) reduces to

$$
\left(\frac{\partial^{2}}{\partial \zeta^{2}}-2 i \frac{\omega}{c} \frac{\partial}{\partial \zeta}-D_{p}\right) \hat{\rho}=\frac{1}{2 \gamma_{\perp 0}^{2}}\left(D_{p}-k^{2}-k_{\perp}^{2}\right)\left(a_{0}^{*} a_{+}+a_{0} a_{-}^{*}\right)
$$

where $D_{p}=\omega^{2} / c^{2}-k_{p}^{2} / \gamma_{\perp 0}$ is the dispersion relation for the plasma wave. With the eikonal approximation $\left|\partial_{\zeta} a_{ \pm}\right| \ll$ $\left|k_{ \pm} a_{ \pm}\right|$, the evolution equations for the daughter waves Eq. (8) reduce to

$$
\begin{aligned}
& \left(D_{+}+2 i \omega_{+} \frac{\partial}{\partial \tau}\right) a_{+}=\frac{k_{p}^{2}}{2 \gamma_{\perp 0}} a_{0} \hat{\rho}, \\
& \left(D_{-}-2 i \omega_{-} \frac{\partial}{\partial \tau}\right) a_{-}^{*}=\frac{k_{p}^{2}}{2 \gamma_{\perp 0}} a_{0}^{*} \hat{\rho},
\end{aligned}
$$

where $D_{ \pm}=\omega_{ \pm}^{2} / c^{2}-k_{ \pm}^{2}-k_{\perp}^{2}-k_{p}^{2} / \gamma_{\perp 0}$ is the dispersion relation for each daughter wave. We take the pump wave to satisfy the dispersion relation $D_{0}=\omega_{0}^{2} / c^{2}-k_{0}^{2}-k_{p}^{2} / \gamma_{\perp 0}=0$. Note that the resonance condition $\varphi_{ \pm}=\varphi_{0} \pm \varphi_{p}$ implies $\omega_{ \pm}=\omega_{0} \pm \omega$ and $k_{ \pm}=k_{0} \pm k$. Without loss of generality, we may take $D_{p}=0$ and $D_{-}=0$, which implies that the plasma wave number is $k \simeq k_{p} / \gamma_{\perp 0}$, assuming $k_{p} / k_{0} \ll 1$ and $\left|k_{\perp} / k_{0}\right| \ll 1$ (i.e., underdense plasma and direct RFS or near-forward RSS). For RFS or near-forward RSS, the anti-Stokes wave is approximately resonant $\left|D_{+} / k_{0}^{2}\right| \simeq 2 k_{\perp}^{2} / k_{0}^{2} \ll 1$.

For definiteness, we will consider a pump laser pulse with a flat-top distribution such that $a_{0}(\zeta)=a_{0}$ for $\zeta \in[-L, 0]$ (i.e., the head of the pulse is located at $\zeta=0$ and the tail of the pulse at $\zeta=-L$ ), and a linear chirp on the pump laser pulse $\varphi_{0}=k_{0} \zeta+\left(\Delta k_{e} / 2\right) \zeta(1+\zeta / L)$. The local wave number is $\partial_{\zeta} \varphi_{0}=k_{0}+\Delta k_{e}(\zeta / L+1 / 2)$, such that $k_{0}$ is the central wave number and $\Delta_{e} \equiv \Delta k_{e} / k_{0}$ is the relative chirp over the FWHM pump laser pulse length. By assuming this form of the pump laser pulse, we are neglecting pump dispersion effects. This is justified since the growth length of the Raman instabilities is much shorter than the dispersive broadening length.

Equations (12) and (13) can be combined, for $k_{p} / k_{0} \ll 1$ and $\left|k_{\perp} / k_{0}\right| \ll 1$, to yield

$$
\frac{\partial^{2}}{\partial \tau^{2}}\left(a_{0}^{*} a_{+}+a_{0} a_{-}^{*}\right)=-\frac{k_{p}^{2}\left|a_{0}\right|^{2}}{4\left(\partial_{\zeta} \varphi_{0}\right)^{2} \gamma_{\perp 0}}\left(k_{\perp}^{2}-2 i k \frac{\partial}{\partial \tau}\right) \hat{\rho},
$$

In the weakly-coupled regime such that $\left|\partial_{\zeta} \hat{\rho}\right| \ll|k \hat{\rho}|$, Eqs. (11) and (14) can be combined to yield

$$
\frac{\partial^{3} \hat{\rho}}{\partial \zeta \partial \tau^{2}}=-i \frac{k_{p}^{3}\left|a_{0}\right|^{2}\left(1+k_{\perp}^{2} / k^{2}\right)}{16 \gamma_{\perp 0}^{5 / 2}\left(\partial_{\zeta} \varphi_{0}\right)^{2}}\left(k_{\perp}^{2}-2 i k \frac{\partial}{\partial \tau}\right) \hat{\rho} .
$$

Equation (15) describes both the direct RFS and near-forward RSS instabilities.

Several regimes of RFS and near-forward RSS can be identified, ${ }^{24-27}$ and, as the instability grows, it passes through these various regimes depending on the value of $|\zeta| / \tau$ and the laser-plasma parameters. In the following sections, we will consider the early time behavior of the Raman instability, where both the Stokes and anti-Stokes waves are approximately resonant. In Sec. III A, we consider plasma wave generation through axial energy transport (i.e., RFS), and, in Sec. III B, we consider plasma wave generation through radial in addition to axial energy transport (i.e., RSS or the self-modulation instability).

\section{A. Raman forward scattering}

In this section we consider plasma wave generation through axial energy transport or RFS. Physically, RFS occurs due to the beating of the incident and scattered light, producing a ponderomotive force which generates a plasma 


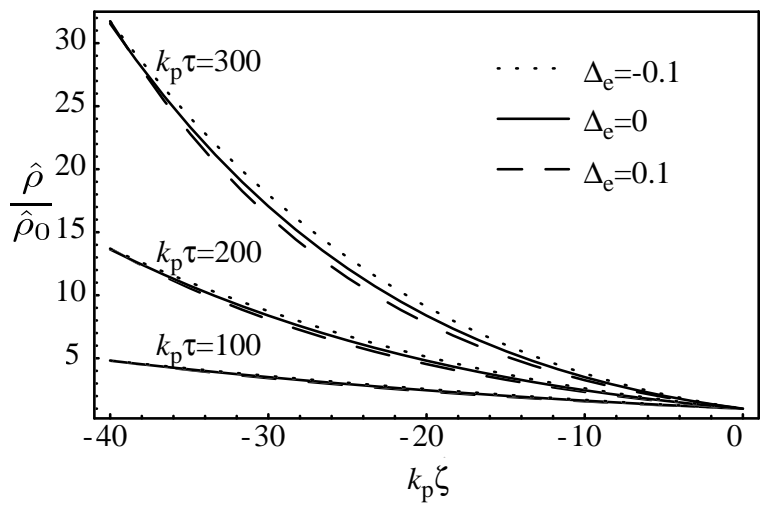

FIG. 4: Growth of plasma wave amplitude $\hat{\rho} / \hat{\rho}_{0}$ due to RFS vs. phase $k_{p} \zeta$ at three times $k_{p} \tau=100, k_{p} \tau=200$, and $k_{p} \tau=300$ for the parameters: $a_{0}=1, k_{0} / k_{p}=10$, and $k_{p} L=40$. Solid line is laser pulse without chirp $\Delta_{e}=0$, dashed line is laser pulse with negative chirp $\Delta_{e}=0.1$, and dotted line is laser pulse with positive chirp $\Delta_{e}=-0.1$.

density modulation at or near the plasma frequency. The plasma density modulation appears as an index of refraction modulation to the incident laser pulse, which results in axial bunching of laser energy, resulting in additional scattering, thereby producing an instability.

In the one-dimensional (1D) limit, where $\left|k_{\perp}^{2} \hat{\rho} /(2 k)\right| \ll\left|\partial_{\tau} \hat{\rho}\right|$ and $\left|k_{\perp} / k\right| \ll 1$, Eq. (15) reduces to

$$
\left(\frac{\partial^{2}}{\partial \zeta \partial \tau}+\Gamma_{4 \text { loc }}^{2}\right) \hat{\rho}=0
$$

where

$$
\Gamma_{4 \mathrm{loc}}(\zeta)=\frac{k_{p}^{2}\left|a_{0}\right|}{\sqrt{8} \gamma_{\perp 0}^{2}\left(\partial_{\zeta} \varphi_{0}\right)}
$$

is the relativistic growth rate for the four-wave resonant RFS instability at the local wave number of the pump laser $\partial_{\zeta} \varphi_{0}$. The solution for the amplitude of the plasma wave potential inside the laser pulse (for $-L \leq \zeta \leq 0$ ) is ${ }^{27}$

$$
\hat{\rho}(\tau, \zeta)=\hat{\rho}_{0} H(\tau) I_{0}\left[2 \Gamma_{4 \mathrm{eff}} \sqrt{\tau|\zeta|}\right]
$$

where $I_{0}$ is the modified Bessel function of zeroth-order. Asymptotically $k_{p}^{2}|\zeta| \tau \gg 1$, the amplitude of the plasma wave grows exponentially as $\hat{\rho} \sim \exp \left[2 \Gamma_{4 \mathrm{eff}} \sqrt{\tau|\zeta|}\right]$. The effective growth rate of the plasma wave due to the four-wave resonant RFS instability is a function of position within the pump laser pulse:

$$
\Gamma_{4 \mathrm{eff}}(\zeta)=\Gamma_{4 \mathrm{loc}}\left[1-\Delta_{e} \frac{|\zeta|}{L}\left(1+\frac{\Delta_{e}}{2}\right)^{-1}\right]^{1 / 2} .
$$

For a positive chirp (i.e., $\Delta_{e}<0$, with red wavelengths at the head and blue wavelengths at the tail of the laser pulse), the RFS growth rate Eq. (19) is greater than the local growth rate throughout the laser pulse. Note that for the unchirped case, $\Delta_{e}=0$, the effective growth rate reduces to $\Gamma_{4}=k_{p}^{2}\left|a_{0}\right| /\left(\sqrt{8} \gamma_{\perp 0}^{2} k_{0}\right)$, the usual nonlinear growth rate for the four-wave resonant RFS instability. ${ }^{28}$ For $\left|\Delta_{e}\right| \ll 1$, the lowest-order correction to the growth rate due to chirp is $\Gamma_{4 \mathrm{eff}} \simeq \Gamma_{4}\left[1-\left(\Delta_{e} / 2\right)(1-|\zeta| / L)\right]$.

Figure 4 shows the growth of the plasma wave amplitude $\hat{\rho} / \hat{\rho}_{0}$ [Eq. (18)] due to the four-wave resonant RFS instability versus phase $k_{p} \zeta$ (location within the pump laser pulse) for the normalized parameters: $a_{0}=1, k_{0} / k_{p}=10$, and $k_{p} L=40$. The figure shows that for positive (negative) chirp $\Delta_{e}<0\left(\Delta_{e}>0\right)$, the growth rate of the four-wave resonant RFS instability is larger (smaller) than for the unchirped case $\left(\Delta_{e}=0\right)$.

As Eq. (18) indicates (and shown in Fig. 4), the growth of the instability is relatively insensitive to the frequency chirp. For example, at the center of the pulse, $\Gamma_{4 \mathrm{eff}}(\zeta=-L / 2)=\Gamma_{4}\left(1+\Delta_{e} / 2\right)^{-1 / 2}$, and the growth rate is changed by only $0.64 \%$ due to a $2.6 \%$ negative chirp over the pump laser pulse (i.e., the experimental bandwidth). Physically this can be understood through the resonance condition for the laser-plasma interaction $\varphi_{ \pm}=\varphi_{0} \pm \varphi_{p}$, which shows that the daughter waves adopt approximately the same local frequency as the pump laser, and therefore the resonant 
excitation of the instability continues as the plasma modulation slips through the pulse. The weak dependence on the sign of the chirp can be understood by considering the local growth rate. The growth of the instability at any location inside the pulse $\zeta$ will be determined by the plasma wave generation from the head to the location $\zeta$. Since the local growth rate [cf. Eq. (17)] is larger at the head for positive chirp (red wavelengths at the head), the enhancement in the growth rate for positive chirp is a result of larger cumulative growth of the plasma density modulation from the head of the pulse to $\zeta$, compared to negative chirp.

These results are valid in the four-wave resonant regime. The $1 \mathrm{D}$ RFS instability will be in the four-wave resonant regime provided $\left|a_{0}\right|^{2}|\zeta| / \tau \gg 8\left(k_{p} / k_{0}\right)^{4}$. For sufficiently long times, this condition will no longer be satisfied, and the RFS instability will transition into the four-wave nonresonant regime. ${ }^{27}$

RFS will dominate RSS provided $\left|\partial_{\tau} \hat{\rho}\right| \gg\left|\left(k_{\perp}^{2} / 2 k\right) \hat{\rho}\right|$. Using the RFS growth rate, this condition reduces to $\left(k_{\perp} / k_{p}\right)^{4} \ll\left[a_{0}^{2} /\left(4 \gamma_{\perp 0}^{5}\right)\right]\left(k_{p} / k_{0}\right)^{2}|\zeta| / \tau$. Therefore, for sufficiently long time or small transverse laser structure $\left(\sim k_{\perp}^{-1}\right)$, the instability will transition into the regime where RSS (the self-modulation instability) will dominate the growth of the plasma wave.

\section{B. Raman sidescatter}

In Sec. III A we considered axial transport of laser energy, which results in modulation of the pump laser at the plasma frequency. For certain laser plasma parameters, the modulation of the laser pulse can be created by radial in addition to axial transport of laser energy via RSS. Physically, the radial transport occurs due to the plasma wave generating periodic regimes of enhanced focusing and defocusing. This leads to bunching of the laser intensity, which drives high phase velocity plasma waves.

In the regime where $\left|\left(k_{\perp}^{2} / 2 k_{0}\right) \hat{\rho}\right| \ll\left|\partial_{\tau} \hat{\rho}\right| \ll\left|\left(k_{\perp}^{2} / 2 k\right) \hat{\rho}\right|$, Eq. (15) reduces to

$$
\left[\frac{\partial^{3}}{\partial \zeta \partial \tau^{2}}+i \Gamma_{\mathrm{RSS}}^{3}\right] \hat{\rho}=0
$$

where

$$
\Gamma_{\mathrm{RSS}}^{3}(\zeta)=\frac{\left|a_{0}\right|^{2} k_{p}^{3} k_{\perp}^{2}}{16 \gamma_{\perp 0}^{5 / 2}\left(\partial_{\zeta} \varphi_{0}\right)^{2}}\left(1+\frac{k_{\perp}^{2} \gamma_{\perp 0}}{k_{p}^{2}}\right)
$$

is the local growth rate of of the RSS instability. Asymptotically Eq. (20) has the solution $\hat{\rho} \sim \hat{\rho}_{0} \exp \left[N_{\mathrm{RSS}}\left(\zeta, \tau ; \Delta_{e}\right)\right]$, where the exponentiation is given by

$$
N_{\mathrm{RSS}}=\frac{3}{8}(\sqrt{3}+i) \tau^{2 / 3}\left[\frac{k_{p}^{3}\left|a_{0}\right|^{2} k_{\perp}^{2}}{\gamma_{\perp 0}^{5 / 2} k_{0}^{2}}\left(1+\frac{k_{\perp}^{2} \gamma_{\perp 0}}{k_{p}^{2}}\right)\right]^{1 / 3}\left(\frac{L}{\Delta_{e}}\right)^{1 / 3}\left\{\left(1+\frac{\Delta_{e}}{2}\right)^{-1}-\left[1+\Delta_{e}\left(\frac{1}{2}+\frac{\zeta}{L}\right)\right]^{-1}\right\}^{1 / 3}
$$

In the limit $a_{0}<1, k_{p}>k_{\perp} \equiv 1 / r_{s}$, and $\Delta_{e}<1$, the exponentiation reduces to

$$
N_{\mathrm{RSS}} \simeq \frac{3(\sqrt{3}+i)}{2}\left(\frac{P}{P_{c}} k_{p}|\zeta|\right)^{1 / 3}\left(\frac{\tau}{Z_{\mathrm{R}}}\right)^{2 / 3}\left[1-\frac{\Delta_{e}}{3}\left(1-\frac{|\zeta|}{L}\right)\right],
$$

where $Z_{\mathrm{R}}=k_{0} r_{s}^{2} / 2$ is the laser Rayleigh length and $P_{c}=k_{p}^{2} a_{0}^{2} r_{s}^{2} / 32$ is the critical power for relativistic self-guiding. For the unchirped case $\Delta_{e}=0$, Eq. (23) reduces to the well-known growth rate for the self-modulation instability. ${ }^{29,30}$ As Eq. (23) indicates, the change in the RSS growth rate due to chirp will be relatively small. For example, at the center of the pulse, $\left|N_{\mathrm{RSS}}\left(-L / 2, \Delta_{e}\right)\right| /\left|N_{\mathrm{RSS}}\left(-L / 2, \Delta_{e}=0\right)\right| \simeq\left(1-\Delta_{e} / 6\right)$, and the growth rate is changed by only $0.43 \%$ due to a $2.6 \%$ negative chirp over the pump laser pulse (i.e., the experimental bandwidth).

As with RFS, the near-forward RSS instability growth rate is larger throughout the pulse for positive chirp than for negative chirp. This is due to the enhanced local growth rate at the head of the pulse for positive chirp (red wavelengths at the head) [cf. Eq. (21)], which produces a larger plasma density modulation. This plasma density modulation slips back through the pulse seeding the RSS.

For RFS, the phase velocity of the excited plasma wave $\beta_{\varphi}$ is approximately the group velocity of the pump laser pulse. Physically this results from the $\pi / 2$ phase shift between the density perturbations and the intensity modulations produced by RFS. For RSS, the density perturbations and the intensity modulations are not $\pi / 2$ out of phase, ${ }^{31}$ which leads to a shift in the phase velocity of the plasma wave. ${ }^{32}$ The phase velocity of the plasma wave generated by RSS is

$$
1-\beta_{\varphi} \simeq \frac{1}{k} \partial_{\tau} \operatorname{Im}\left(N_{\mathrm{RSS}}\right) \simeq \frac{1}{2 k_{p} Z_{\mathrm{R}}}\left[\frac{P}{P_{c}} \frac{k_{p}|\zeta|}{\left(\tau / Z_{\mathrm{R}}\right)}\right]^{1 / 3}\left[1-\frac{\Delta_{e}}{3}\left(1-\frac{|\zeta|}{L}\right)\right]
$$


which indicates that the phase velocity of the plasma wave generated by RSS decreases in time. ${ }^{30}$

In deriving Eq. (15) it was assumed that $\left|\left(k_{\perp}^{2} / 2 k_{0}\right) \hat{\rho}\right| \ll\left|\partial_{\tau} \hat{\rho}\right|$, which is equivalent to assuming that both the Stokes and anti-Stokes waves are resonant. This condition can be expresses as $\left(\tau / Z_{\mathrm{R}}\right) \gamma_{\perp 0}^{7 / 2} / 2^{6} \ll\left(P / P_{c}\right) k_{p}|\zeta|$, which indicates that for sufficiently long propagation times, the Raman instability will transition into the three-wave regime where the anti-Stokes wave is no longer resonant.

\section{PULSE SHAPE SEEDING OF RAMAN INSTABILITIES}

In Sec. III we have shown that the frequency chirp has a relatively minor effect on the growth rates of the Raman instabilities which drive high phase velocity plasma waves, e.g., the growth rates were changed by $\lesssim 1 \%$ for the experimental frequency chirp $(\sim 2.6 \%)$. As described in Sec. II, the presence of higher-order phase dispersive terms in the optical transport line will modify the temporal envelope of the pulse, resulting in skewed laser pulses. In this section, we consider the effect of pulse shape (temporal distribution) on the generation of plasma waves.

The Raman instability is initially seeded by plasma density fluctuations or pump laser intensity fluctuations which contain Fourier components at the relativistic plasma frequency $k=k_{p} / \gamma_{\perp 0}^{1 / 2}$. For example the seeding of the RFS may be generated by thermal fluctuations in the plasma, ionization-induced plasma waves (owing to a time-varying dielectric), ${ }^{33,34}$ or ponderomotively-excited plasma waves (owing to the laser intensity gradient). ${ }^{10,35}$

Experiments often will use the same pump laser pulse which undergoes self-modulation to create the plasma through ionization of a gas. Typically photoionization will occur very early in the head of the laser pulse, where the laser electric field becomes sufficiently intense such that the rate of ionization is maximum. ${ }^{36}$ This will create a plasma density front moving with the laser. The amplitude of the ionization-induced plasma waves created by this ionization front will be approximately $\hat{\rho}_{0} \sim a_{0}^{2}\left(\zeta_{\text {ionz }}\right) / 4$, where $\zeta_{\text {ionz }}$ is the location in the pump laser pulse where the rate of ionization is maximum. Note that the ionization location $\zeta_{\text {ionz }}$ is weakly dependent on the chirp. For typical laser experimental parameters, the shift in the ionization location due to chirp is less than a laser wavelength $k_{0} \zeta_{\text {ionz }} \lesssim 1$, and therefore there will be little asymmetry in the seeding of Raman scattering owing to a frequency-chirp shift in ionization location.

The amplitude of the ponderomotively-excited plasma waves scales as $\hat{\rho}_{0} \sim k_{p}^{-2} \nabla^{2} a_{0}^{2} \sim a_{0}^{2} /\left(k_{p} L_{\text {rise }}\right)^{2}$ for $k_{p} L_{\text {rise }}>1$, where the gradient in the rise in the laser pulse intensity scales as $\sim 1 / L_{\text {rise }}$. For short laser pulses, the ponderomotively excited plasma wave will typically dominate other sources for seeding the Raman instability. For example, consider Fig. 5, which shows simulation results from a nonparaxial envelope code ${ }^{37}$ of the laser pulse self-modulation. Figure 5 shows initial (a) positively-skewed and (c) negatively-skewed laser pulses and ponderomotively-excited wakes, and (b) positively-skewed and (d) negatively-skewed laser pulses and resulting plasma waves after propagating one Rayleigh range, for the laser-plasma parameters: $k_{0} / k_{p}=6, k_{p} r_{s}=12,76$ fs FWHM pulse durations, and $a_{0}=1 / 3$. Initially the positively-skewed pulse excites several plasma oscillations within the pulse envelope [Fig. 5(a)] that seed the laser self-modulation [Fig. 5(b)]. Plasma oscillations are not excited within the pulse envelope of the negatively-skewed pulse [Fig. 5(c)], and therefore the instability is not strongly-seeded and self-modulation does not occur [Fig. 5(d)].

To evaluate the ponderomotively-excited seed, the initial plasma response was calculated for the experimental temporal laser profiles using the 1D nonlinear quasi-static fluid equations. ${ }^{1}$ The evolution equation for the quasistatic fluid perturbation is

$$
\frac{\partial^{2} \phi}{\partial \zeta^{2}}=\frac{k_{p}^{2}}{2}\left[\frac{\gamma_{\perp 0}^{2}(\zeta)}{(1+\phi)^{2}}-1\right] .
$$

Here $\phi=\gamma n_{0} / n-1$ is the quasi-static potential, and $\gamma_{\perp 0}^{2}(\zeta)=1+\left|a_{0}\right|^{2}(\zeta) / 2$, where $\left|a_{0}\right|(\zeta)$ is the slowly-varying envelope of the pump laser pulse. Figure 6(a) shows the plasma wave potential perturbation $\delta \phi=\phi-\left(\gamma_{\perp 0}-1\right)$ ponderomotively-excited by the steep $a_{+}(\zeta)$ and gentle $a_{-}(\zeta)$ rising experimental laser pulse profiles with 76 fs FWHM duration. A larger wake is excited within the laser pulse for the case of the steep rising (positively-chirped) laser pulse than for the gentle rising (negatively-chirped) laser pulse. Figure 6(b) shows the ratio of the Fourier components [evaluated at the relativistic plasma frequency at peak intensity $\Omega_{p}=k_{p} c /\left.\gamma_{\perp 0}^{1 / 2}\right|_{\text {peak }}$ ] of the ponderomotively-excited seeds $\Lambda=\left.\left(\mathcal{F}\left[a_{+} \delta \phi_{+}\right] / \mathcal{F}\left[a_{-} \delta \phi_{-}\right]\right)\right|_{\Omega_{p}}$ for the steep and gentle rising pulses from Fig. 6 (a) for varying density. The ratio $\Lambda$ is a figure of merit for the asymmetry in the Raman instability seeds for positive and negative skew. Figure 6(b) shows a large asymmetry in the Raman instability seed when $k_{p} L \sim 10$. Also shown in Fig. 6(b) is the maximum amplitude of the seed $\left[a_{+} \delta \phi_{+}(\zeta)\right]_{\max }$ which decreases with increasing density or pulse duration. Hence, for high density or long pulse duration, the pulse envelope contribution to the seeding of RFS is small.

For case 1 of the experimental results discussed in Sec. II (cf. Fig. 3), the laser is at high intensity throughout the whole plasma; it enters at high intensity and remains focused due to relativistic self-guiding. As the pulse encounters 

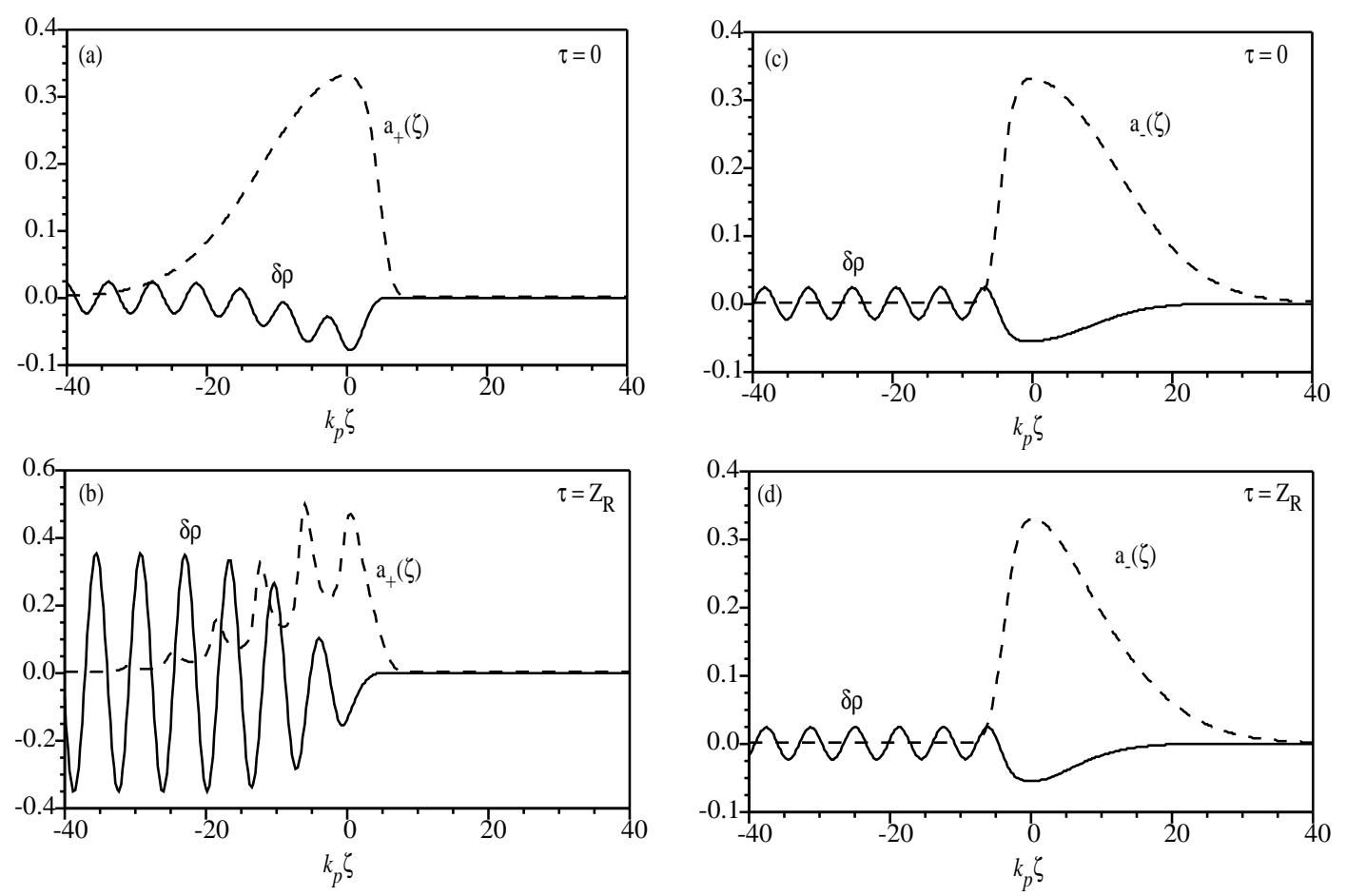

FIG. 5: (a) Initial $(\tau=0)$ positively-skewed laser pulse $a_{+}$and ponderomotively-excited wake $\delta \rho$ and (b) positively-skewed laser pulse and resulting plasma wave after propagating one Rayleigh range $\left(\tau=Z_{\mathrm{R}}\right)$. (c) Initial $(\tau=0)$ negatively-skewed laser pulse $a_{-}$and ponderomotively-excited wake $\delta \rho$ and $(\mathrm{d})$ negatively-skewed laser pulse and resulting plasma wave after propagating one Rayleigh range $\left(\tau=Z_{\mathrm{R}}\right)$. Laser-plasma simulation parameters: $k_{0} / k_{p}=6, k_{p} r_{s}=12,76$ fs $\mathrm{FWHM}$ pulse durations, and $a_{0}=1 / 3$.

the low density plasma in the front of the gas jet profile, $k_{p} L \sim 10$ and the fast rise time for the positive chirped pulse is able to excite large plasma waves, resulting in a larger seed for the Raman instability. For the slow rise time pulse (negative chirp), the response is weaker and lower amplitude plasma waves ensue. The asymmetry in redshifted sidebands observed in the experiment is also consistent with enhanced Raman scattering for fast rise time (positive chirp) pulses. ${ }^{20} \mathrm{~A}$ larger seed leads to electron trapping earlier in the laser-plasma interaction, resulting in larger electron yield.

When the laser pulse was focused deeper into the gas jet (case 2 of Fig. 3), it traverses much of the lower density gas at low intensity. As can be seen in Fig. 6(b), in the high density region where $k_{p} L \gtrsim 20$, seeding of the plasma wave due to envelope effects is much less sensitive to pulse asymmetries, i.e., $\Lambda \sim 1$. Therefore the maximum wave amplitude (electron yield) occurs for shorter laser pulses at higher intensities, i.e., near the normal compressor zero, as shown in Fig. 3.

\section{SUMMARY AND CONCLUSIONS}

In this work, we have presented experimental and theoretical studies of the effect of pulse shape and chirp asymmetries in the SM-LWFA.

Higher-order phase dispersive terms associated with optical components in the laser system can cause pulse shape asymmetries with fast rises of the order of the plasma period (positively-skewed), which are more unstable to RFS and self-modulation instabilities. These instabilities drive high phase velocity plasma waves, resulting in larger electron yield in SM-LWFA experiments. This was verified experimentally (cf. case 1 of Fig. 3 ) by focusing the laser into a plasma density such that $k_{p} L \sim 10$, where asymmetric seeding of the Raman instability by the pulse envelopes will strongly affect plasma wave excitation [cf. Fig. 6(b)].

Although a frequency chirp was found analytically to increase the growth rate for RFS and near-forward RSS for a positive chirp (i.e., red wavelengths at the head of the pulse), this enhancement is a relatively minor effect and of minimal importance for the experimental parameters. For example, the growth rates were changed by $\lesssim 1 \%$ for the experimental frequency chirp $(\sim 2.6 \%)$. This was verified experimentally (cf. case 2 of Fig. 3$)$ by focusing the laser 

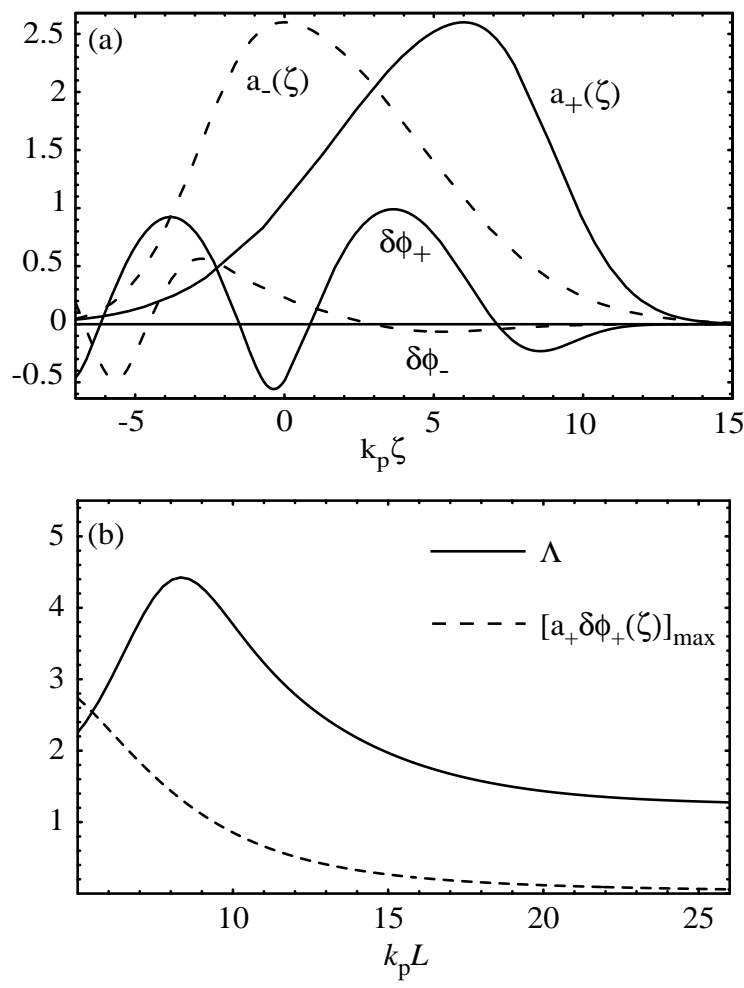

FIG. 6: (a) Plasma wave potential $\delta \phi_{ \pm}$excited by pulse envelope $a_{ \pm}(\zeta)$ for positive (solid curves) and negative (dashed curves) chirped pulses with $k_{p} L=6$ for $2 \times 10^{18} \mathrm{~cm}^{-3}$ density. (b) Solid curve is the ratio $\Lambda=\left.\left(\mathcal{F}\left[a_{+} \delta \phi_{+}\right] / \mathcal{F}\left[a_{-} \delta \phi_{-}\right]\right)\right|_{\Omega_{p}}$ of the Fourier components (evaluated at relativistic plasma frequency for peak intensity $\left.\Omega_{p}\right)$ of the Raman scattering seeds $\left(a_{ \pm} \delta \phi_{ \pm}\right)$with pulse shapes of (a) as a function of density. Dashed curve is the peak seed of the positive chirped pulse $\left[a_{+} \delta \phi_{+}(\zeta)\right]_{\text {max }}$.

into a high plasma density where we expect little asymmetric seeding by the pulse envelopes, isolating the effect of the frequency chirp. In this case, no asymmetry in electron yield was observed.

These results indicate that pulse shaping provides a method of controlling plasma instabilities and optimizing the performance of laser-plasma devices. Although the SM-LWFA relies on an instability, the plasma wave amplitude can be controlled by controlling the seed. Since the ponderomotively-excited wake dominates other noise sources in this parameter regime, pulse shaping allows the experimenter a controllable and reproducible seed for the instability. This offers a method for minimizing shot-to-shot output fluctuations in electron yield and other beam properties in the SM-LWFA.

\section{Acknowledgments}

This work was supported by the U.S. Department of Energy under Contract No. DE-AC03-76SF0098. The authors acknowledge useful conversations with G. Fubiani and R. Trines.

* Paper KI1 5, Bull. Am. Phys. Soc. 47, 181 (2002).

$\dagger$ CBSchroeder@lbl.gov; Invited speaker.

$¥$ Also at Technische Universiteit Eindhoven, the Netherlands.

1 E. Esarey, P. Sprangle, J. Krall, and A. Ting, IEEE Trans. Plasma Sci. 24, 252 (1996).

2 E. Esarey, S. K. Ride, and P. Sprangle, Phys. Rev. E 48, 3003 (1993).

${ }^{3}$ P. Amendt, D. C. Eder, and S. C. Wilks, Phys. Rev. Lett. 66, 2589 (1991).

${ }^{4}$ M. Tabak, J. Hammer, M. E. Glinsky, W. L. Kruer, S. C. Wilks, J. Woodworth, E. M. Campbell, M. D. Perry, and R. J. Mason, Phys. Plasmas 1, 1626 (1994).

${ }^{5}$ G. Laval, R. Pellat, D. Pesme, A. Ramani, M. N. Rosenbluth, and E. A. Williams, Phys. Fluids 20, 2049 (1977).

${ }^{6}$ P. N. Guzdar, C. S. Liu, and R. H. Lehmberg, Phys. Fluids B 3, 2882 (1991). 
7 R. H. Lehmberg, A. J. Schmitt, and S. E. Bodner, J. Appl. Phys. 62, 2680 (1987).

8 E. S. Dodd and D. Umstadter, Phys. Plasma 8, 3531 (2001).

9 V. I. Berezhiani and I. G. Murusidze, Physica Scripta 45, 87 (1992).

10 J. Krall, E. Esarey, P. Sprangle, and G. Joyce, Phys. Plasmas 1, 1738 (1994).

11 W. L. Kruer, The Physics of Laser Plasma Interactions (Addison-Wesley, Redwood City, CA, 1988).

12 A. Modena, Z. Najmudin, A. E. Dangor, C. E. Clayton, K. A. Marsh, C. Joshi, V. Malka, C. B. Darrow, C. Danson, D. Neely, et al., Nature 377, 606 (1995).

13 D. Umstadter, S.-Y. Chen, A. Maksimchuk, G. Mourou, and R. Wagner, Science 273, 472 (1996).

14 A. Ting, C. I. Moore, K. Krushelnick, C. Manka, E. Esarey, P. Sprangle, R. Hubbard, H. R. Burris, R. Fischer, and M. Baine, Phys. Plasmas 4, 1889 (1997).

15 C. Gahn, G. D. Tsakiris, A. Pukhov, J. Meyer-ter-Vehn, G. Pretzler, P. Thirolf, D. Habs, and K. J. Witte, Phys. Rev. Lett. 83, 4772 (1999).

${ }^{16}$ V. Malka, J. Faure, J. R. Marquès, F. Amiranoff, J. P. Rousseau, S. Ranc, J. P. Chambaret, Z. Najmudin, B. Walton, P. Mora, et al., Phys. Plasmas 8, 2605 (2001).

17 W. P. Leemans, D. Rodgers, P. E. Catravas, C. G. R. Geddes, G. Fubiani, E. Esarey, B. A. Shadwick, R. Donahue, and A. Smith, Phys. Plasmas 8, 2510 (2001).

18 J. Faure, J.-R. Marquès, V. Malka, F. Amiranoff, Z. Najmudin, B. Walton, J.-P. Rousseau, S. Ranc, A. Solodov, and P. Mora, Phys. Rev. E 63, 065401 (2001).

19 T.-W. Yau, C.-J. Hsu, H.-H. Chu, Y.-H. Chen, C.-H. Lee, J. Wang, and S.-Y. Chen, Phys. Plasmas 9, 391 (2002).

${ }^{20}$ W. P. Leemans, P. Catravas, E. Esarey, C. G. R. Geddes, C. Toth, R. Trines, C. B. Schroeder, B. A. Shadwick, J. van Tilborg, and J. Faure, Phys. Rev. Lett. 89, 174802 (2002).

${ }^{21}$ C. Tóth, J. de Groot, J. van Tilborg, C. Geddes, J. Faure, P. Catravas, C. Schroeder, B. Shadwick, E. Esarey, and W. Leemans, in Proceedings of the 13th International Conference on Ultrafast Phenomena, edited by D. R. Miller, M. M. Murnane, N. F. Scherer, and A. M. Weiner (Springer-Verlag, NY, 2002), vol. 71.

22 E. Esarey, P. Sprangle, J. Krall, and A. Ting, IEEE J. Quantum Electron. 33, 1879 (1997).

23 X. L. Chen and R. N. Sudan, Phys. Fluids B 5, 1336 (1993).

24 T. M. Antonsen, Jr. and P. Mora, Phys. Rev. Lett. 69, 2204 (1992).

25 T. M. Antonsen, Jr. and P. Mora, Phys. Fluids B 5, 1440 (1993).

26 C. D. Decker, W. B. Mori, T. Katsouleas, and D. E. Hinkel, Phys. Plasmas 3, 1360 (1996).

27 C. B. Schroeder, E. Esarey, B. A. Shadwick, and W. P. Leemans, Phys. Plasmas 10, 285 (2003).

28 W. B. Mori, C. D. Decker, D. E. Hinkel, and T. Katsouleas, Phys. Rev. Lett. 72, 1482 (1994).

29 E. Esarey, J. Krall, and P. Sprangle, Phys. Rev. Lett. 72, 2887 (1994).

30 N. E. Andreev, V. I. Kirsanov, L. M. Gorbunov, and A. S. Sakharov, IEEE Trans. Plasma Sci. 24, 363 (1996).

31 W. B. Mori, IEEE J. Quantum Electron. 33, 1942 (1997).

32 W. P. Leemans, C. W. Siders, E. Esarey, N. E. Andreev, G. Shvets, and W. B. Mori, IEEE Trans. Plasma Sci. 24, 331 (1996).

33 W. B. Mori and T. Katsouleas, Phys. Rev. Lett. 69, 3495 (1992).

34 D. L. Fisher and T. Tajima, Phys. Rev. E 53, 1844 (1996).

35 P. Sprangle, E. Esarey, A. Ting, and G. Joyce, Appl. Phys. Lett. 53, 2146 (1988).

${ }^{36}$ P. Sprangle, E. Esarey, and J. Krall, Phys. Rev. E 54, 4211 (1996).

37 E. Esarey, C. B. Schroeder, B. A. Shadwick, J. S. Wurtele, and W. P. Leemans, Phys. Rev. Lett. 84, 3081 (2000). 